\title{
Heart rate variation analysis may not effectively detect sleep apnoeas in heart failure
}

\section{To the Editors:}

In a recent issue of the European Respiratory Journal, VAZIR et al. [1] demonstrated that spectral analysis of heart rate variations (HRV) may be useful as a "rule-out test" for sleep-disordered breathing (SDB) in patients with mild-to-moderate congestive heart failure (CHF). This is an interesting approach to the diagnosis of SDB in CHF patients without sleepiness. As CHF patients with Cheyne-Stokes respiration (CSR)/central sleep apnoea (CSA) are often asymptomatic, this noninvasive approach may be effective to rule out SDB in patients. However, we feel that there are a number of problems with the article by VAZIR et al. [1].

First, HRV analysis cannot be applied in patients with arrhythmia, including atrial fibrillation (AF). In a group of patients with $\mathrm{CHF}$, it has been demonstrated that there is a considerable association between AF and CSA [2]. Thus, AF may be a feature of CHF with CSR/CSA. As AF is very common in CHF with CSR/CSA, it is difficult for HRV analysis alone to rule out the majority of patients with both AF and SDB. Furthermore, paroxysmal AF (PAF) can occur during the night in patients with sleep apnoea and in those of increased age [3]. The presence of PAF episodes and frequent ventricular ectopy may impair the analysis of HRV in patients with $\mathrm{CHF}$.

Secondly, there is the problem of the causal relationship between AF and CSR/CSA. It has been suggested that the risk factors for CSR/CSA are male sex, AF, aged >60 yrs and hypocapnia. AF may therefore be a causal factor for CSR/CSA. In this scenario, $\mathrm{CHF}$ patients with $\mathrm{AF}$ have a high risk for CSR/CSA. In addition, treatment of arrhythmia with atrial overdrive pacing (AOP) may also have some beneficial effects on sleep apnoea itself [4]. Although obstructive sleep apnoea syndrome predisposes to clinically significant cardiac rhythm disturbances that can be successfully controlled by nasal continuous positive airway pressure, AOP has no significant effect on the respiratory variables, including the apnoea/ hypopnoea index [5]. Therefore, the causal relationships between AF and CSR/CSA are not simple.

Thirdly, CHF patients are often treated with antihypertensive agents. These agents also have some effect on HRV results. It has been reported that a sympathetic activation during the day and a decrease in parasympathetic activity during the night after therapy with a calcium channel blockade, amlodipine, correlated with increases in plasma noradrenaline (NA). In contrast, therapy with an angiotensin II receptor blocker, telmisartan, significantly increased parasympathetic activity without changes in NA during the night and day [6]. It has also been suggested that treatment with a nonselective $\beta$-adrenergic blocking agent, carvedilol, in CHF restores both autonomic balance and the ability to increase reflex vagal activity [7]. Thus, the treatment strategy for CHF with antihypertensive agents has a great impact on HRV analysis.
Autonomic disturbances and central nervous system abnormality also cause SDB and cardiac dysfunction [8]. Patients with neurological disorders and CHF may not undergo HRV analysis.

The large number of CHF patients cannot all be examined in sleep laboratories, so alternative methods of screening for SDB may be required for $\mathrm{CHF}$ patients in AF. Although pulse oximetry may underestimate nondesaturated CSA, it still has an advantage over HRV monitoring in terms of convenient and widely available methods. Thus, CHF patients with negative results in SDB judged by pulse oximetry should be analysed with HRV.

In two previous studies $[9,10]$, sleep apnoea patients had markedly decreased heart rate variability and increased blood pressure variability. These variability abnormalities are characteristic of patients with hypertension. The abnormalities in cardiovascular variability may exaggerate ventricular function and contribute to development of AF and other cardiovascular diseases $[9,10]$. Although cardiovascular variability and HRV abnormality are features of $\mathrm{CHF}$ with SDB, the clinical significance of HRV analysis for screening SDB may be limited.

Because an ideal screening test should have a high sensitivity with a reasonable specificity, we believe that the conclusion drawn by VAZIR et al. [1] should be changed to: "spectral analysis of heart rate variations may be useful as a noninvasive rule-out test for sleep-disordered breathing in patients with congestive heart failure without arrhythmia."

\section{S. Teramoto*, H. Kume*, , Y. Yamaguchi*, H. Yamamoto*, M. Ishii*, T. Ishii* and Y. Ouchi* \\ *Dept of Geriatric Medicine, and "Dept of Urology, Graduate School of Medicine, University of Tokyo, Tokyo, Japan.}

\section{REFERENCES}

1 Vazir A, Dayer M, Hastings PC, et al. Can heart rate variation rule out sleep-disordered breathing in heart failure? Eur Respir J 2006; 27: 571-577.

2 Leung RS, Huber MA, Rogge T, Maimon N, Chiu KL, Bradley TD. Association between atrial fibrillation and central sleep apnea. Sleep 2005; 28: 1543-1546.

3 Schulz R, Eisele HJ, Seeger W. Nocturnal atrial fibrillation in a patient with obstructive sleep apnoea. Thorax 2005; 60: 174.

4 Garrigue S, Bordier P, Jais $\mathrm{P}$, et al. Benefit of atrial pacing in sleep apnea syndrome. N Engl J Med 2002; 346: 404-412.

5 Simantirakis EN, Schiza SE, Chrysostomakis SI, et al. Atrial overdrive pacing for the obstructive sleep apnea-hypopnea syndrome. N Engl J Med 2005; 353: 2568-2577.

6 Karas M, Lacourciere Y, LeBlanc AR, et al. Effect of the renin-angiotensin system or calcium channel blockade on the circadian variation of heart rate variability, blood 
pressure and circulating catecholamines in hypertensive patients. J Hypertens 2005; 23: 1251-1260.

7 Mortara A, La Rovere MT, Pinna GD, Maestri R, Capomolla S, Cobelli F. Reversal of deteriorated fractal behavior of heart rate variability by beta-blocker therapy in patients with advanced congestive heart failure. $J$ Cardiovasc Electrophysiol 2001; 12: 26-32.

8 Teramoto S, Ishii T, Matsuse T. Central sleep apnoeas in patients with Charcot-Marie-Tooth disease. Lancet 2001; 358: 70-71.

9 Teramoto S, Kume H, Matsuse T. Ambulatory blood pressure after sleep apnoea treatment. Lancet 2002; 360: 341.

10 Teramoto S, Matsuse T, Ouchi Y. Does the altered cardiovascular variability in association with obstructive sleep apnea contribute to development of cardiovascular disease in patients with obstructive sleep apnea syndrome? Circulation 1999; 100: e136-e137.

\section{DOI: 10.1183/09031936.06.00044106}

From the authors:

We greatly appreciate the comments made by S. Teramoto and co-workers. In our study [1] we suggested that spectral analysis of heart rate in patients with mild-to-moderate heart failure could be of value in ruling out severe sleep-disordered breathing (SDB), and its use could reduce the burden on sleep laboratories screening for SDB in the heart failure population. However, we stressed that further prospective validation was required and discussed in depth the limitations of spectral analysis of heart rate variability (HRV) as a rule-out test for SDB in heart failure. The key limitation is that it cannot be applied to heart failure patients with atrial fibrillation (AF), or those with extensive pacing or excessive ventricular ectopy. Therefore, these groups of patients were excluded for analysis of HRV in our study. Thus, we agree with S. Teramoto and coworkers that a more precise conclusion would be: "spectral analysis of heart rate variation is useful as a rule-out test for SDB in patients with heart failure without significant arrhythmia".

The major advantage of HRV analysis is that it can be used within the cardiology setting, where cardiologists are using a tool that they are much more familiar with than pulse oximetry. Furthermore, heart failure patients who have already undergone Holter monitoring to assess for potential arrhythmias could also have their Holter monitoring analysed for SDB. As we discussed in our paper, the combination of pulse oximetry together with HRV may be a stronger, more comprehensive screening tool for SDB as patients who are paced or who have arrhythmias can be screened for SDB.

We indicated that the presence of SDB is likely to be high in heart failure patients with AF [2], and as HRV analysis cannot be applied to these patients we have suggested that these individuals should be assessed for SDB using pulse oximetry.

S. Teramoto and colleagues raise the issue of the possible effects of medication on the percentage very low frequency index (\%VLFI) component of spectral analysis of HRV. There are published data on the effects of medication on other aspects of HRV; however, data on the effects of medication on $\%$ VLFI are lacking. In our population of heart failure patients, all patients who underwent HRV analysis were taking angiotensin-converting enzymes or angiotenesin II receptor blocker, and $72 \%$ were also taking $\beta$-blocker, $12 \%$ digoxin and $32 \%$ diuretics. Thus, the results from our study also include the possible effects of medications on \%VLFI. Furthermore, ROCHE and co-workers $[3,4]$ published data on \%VLFI within patients screened for obstructive sleep apnoea, and a significant number of these patients were on various cardiac medications.

S. Teramoto and co-workers also raise the point that heart failure patients with neurological comorbidity may have abnormalities in the autonomic and central nervous system, which could affect heart rate variability. They suggest that such patients should not undergo heart rate variability analysis. In our study, heart failure patients did not have neurological deficits, so findings cannot be extrapolated to heart failure patients with concurrent neurological deficit. The effect of the presence of abnormal neurology on percentage very low frequency index is unclear and further investigation is required.

\section{A. Vazir*, M.J. Morrell ${ }^{\#}$ and A.K. Simonds ${ }^{\#}$}

*Dept of Cardiac Medicine and "Academic and Clinical Unit of Sleep and Breathing, Royal Brompton Hospital, Imperial College, London, UK.

\section{REFERENCES}

1 Vazir A, Dayer M, Hastings PC, et al. Can heart rate variation rule out sleep-disordered breathing in heart failure? Eur Respir J 2006; 27: 571-577.

2 Leung RS, Huber MA, Rogge T, Maimon N, Chiu KL, Bradley TD. Association between atrial fibrillation and central sleep apnea. Sleep 2005; 28: 1543-1546.

3 Roche F, Duverney D, Court-Fortune I, et al. Cardiac interbeat interval increment for the identification of obstructive sleep apnea. Pacing Clin Electrophysiol 2002; 25: 1192-1199.

4 Roche F, Xuong AN, Court-Fortune I, et al. Relationship among the severity of sleep apnea syndrome, cardiac arrhythmias, and autonomic imbalance. Pacing Clin Electrophysiol 2003; 26: 669-677.

DOI: $10.1183 / 09031936.06 .00054806$ 